\title{
EchoGéo
}

29 | 2014

The Political Ecology of Conservation and

Development Territories

\section{To be or not to be}

Jean-Louis Chaléard

\section{(2) OpenEdition}

1 Journals

Electronic version

URL: https://journals.openedition.org/echogeo/14043

DOI: $10.4000 /$ echogeo.14043

ISSN: 1963-1197

Publisher

Pôle de recherche pour l'organisation et la diffusion de l'information géographique (CNRS UMR 8586)

\section{Electronic reference}

Jean-Louis Chaléard, "To be or not to be", EchoGéo [Online], 29 | 2014, Online since 18 November 2014, connection on 01 August 2021. URL: http://journals.openedition.org/echogeo/14043 ; DOI: https:// doi.org/10.4000/echogeo.14043

This text was automatically generated on 1 August 2021.

EchoGéo est mis à disposition selon les termes de la licence Creative Commons Attribution - Pas d'Utilisation Commerciale - Pas de Modification 4.0 International (CC BY-NC-ND) 


\title{
To be or not to be
}

\author{
Jean-Louis Chaléard
}

1 En juillet 2014 a eu lieu à Paris un forum organisé par le CNFG (Comité National de Géographie Français) qui portait sur «Les langues de la diffusion scientifique ». Le présent numéro s'en fait l'écho dans la rubrique Sur le métier. N. Lemarchand et A. Le Blanc font un compte rendu des débats qui ont eu lieu. La question est d'importance. L'anglais est devenu la langue presque exclusive de diffusion des résultats scientifiques à l'échelle internationale. On peut s'en réjouir, parce que l'usage d'un langage commun facilite la transmission des connaissances et favorise les discussions au sein du monde de la recherche. L'anglais est en passe d'avoir un peu le même rôle que le latin pour les clercs au Moyen-Âge. Mais on peut aussi le regretter dans la mesure où cette hégémonie fait perdre une richesse culturelle immense, le génie propre à chaque idiome, qui permet d'approcher, en sciences sociales, les réalités à partir d'une culture et de mots différents. Ces réflexions débouchent sur le débat des conditions de la production scientifique elle-même: diversité des démarches ou science globale? Ces questions ont été discutées lors du forum du CNFG et concernent au premier chef Échogéo, puisqu'il s'agit pour nous de diffuser les résultats de recherches en sciences sociales, en essayant de toucher un public le plus large possible, au-delà des frontières de l'hexagone. Nous en avons discuté dans des numéros précédents. Et c'est pourquoi notre revue, francophone, a déjà accueilli des articles en anglais, outil principal de diffusion des savoirs à l'échelle internationale, et en espagnol, langue parlée dans de nombreux pays.

2 Dans son introduction à la rubrique Sur le métier, Alexis Sierra relève les "mots " empruntés aux langues étrangères dans la géographie française. Ils sont nombreux. On pourrait y inclure l'expression "Political Ecology», plus souvent employée en français dans les sciences sociales que sa traduction littérale (Écologie politique), qui n'a d'ailleurs pas exactement la même signification, car elle renvoie plus directement à un courant politique.

3 La volonté de rompre les barrières linguistiques se traduit aussi dans ce numéro par la construction du dossier de la rubrique Sur le champ, "La régulation par la territorialisation: la Political Ecology de la conservation et du développement des 
territoires ", qui associe à sa mise en œuvre deux chercheurs, Thomas J. Bassett et Denis Gautier, l'un américain, l'autre français, avec des articles en français et en anglais.

4 La Political Ecology est un courant largement développé dans le monde anglo-saxon et dont le succès récent en France s'est traduit par toute une série de publications. Le présent dossier essaie d'enrichir un corpus déjà abondant en mettant la focale sur la dimension territoriale des processus et des questions qui se posent. Il est l'occasion de faire le point sur (ou d'aborder) des notions comme "territoire", "territorialité ", "territorialisation ", à partir d'auteurs anglo-saxons et français. Il met ainsi en évidence un certain nombre de processus de territorialisation en rapport avec le développement et/ou la préservation de l'environnement, que ce soit à partir d'analyses textuelles, de politiques mises en place ou de pratiques des acteurs. Le texte de F. Pochet mène une approche des discours, largement inspirée de Foucault, sur la conservation au Bénin depuis le début $\mathrm{du} \mathrm{XX}^{\mathrm{e}}$ siècle, et montre que malgré les changements, deux éléments demeurent récurrents : l'argument économique qui guide les choix de politiques et la marginalisation des populations pauvres. La territorialisation est le fait de multiples acteurs aux intérêts variables selon les moments et les lieux. L'encadrement des plantations d'eucalyptus par les autorités à Addis Abeda, étudié par D. Ayerbe, révèle à la fois les changements dans l'intérêt porté à l'arbre et comment l'argumentation écologiste peut être instrumentalisée par les autorités au détriment des pratiques populaires. Au contraire, dans un autre contexte, N. List met en évidence, à propos de Pikine et des agriculteurs locaux, les convergences entre groupes d'acteurs, dans ce qu'elle appelle des «alliances territoriales». S. Audouin et A. Gonin montrent comment les dynamiques territoriales actuelles, dans le Sud du Burkina Faso, résultent à la fois d'incitations anciennes de l'État, d'initiatives autochtones, de dynamiques de migrants qui saisissent les opportunités du marché international (ici la hausse des prix de l'anacarde). Pour terminer, F. Chamara-Huff rappelle opportunément que les questions se posent aussi du côté des mers et océans, longtemps vus comme des espaces non territorialisés. Les aires maritimes protégées révèlent différents territoires, produits par des acteurs variés, qui ne sont pas réductibles à la seule juridiction de l'État. Les articles montrent ainsi la diversité des intervenants, du «haut» et du «bas» comme du public et du privé, de leurs motivations et de leurs relations, faites d'oppositions ou d'alliances selon les cas, dans la production de nouvelles configurations territoriale, à partir des processus d'accès aux ressources, à leur contrôle, leur gestion.

5 Si les deux articles de la rubrique Sur l'image ne concernent pas directement la Political ecology, ils abordent néanmoins des questions touchant à l'environnement et soulevés par ce champ d'investigation. Les deux textes portent sur des outils d'analyse autant que sur les résultats eux-mêmes (cartes, photographies, etc.), les documents commentés permettant d'approcher des phénomènes à étudier. Le premier texte vise à présenter la problématique de la pollution de l'air sous l'influence de phénomènes météorologiques d'échelle locale, à l'aide de photographies et schémas dans les Alpes maritimes, région littorale très urbanisée. Le second texte porte sur le recours à la carte pour tenter de démêler l'écheveau des facteurs contribuant à la mise en valeur des biens naturels et culturels du bassin d'Arcachon. La carte présentée par les auteurs doit être un outil de réflexion et de communication sur les enjeux de la 
patrimonialisation dans le bassin. Elle débouche sur l'intérêt d'une étude à partir de la carte et sur une réflexion plus large sur la préservation du patrimoine côtier.

6 La rubrique Sur le vif s'est enrichie de deux nouveaux textes sur l'Ukraine. Ils ont certes peu à voir avec les thèmes principaux de la présente livraison d'Échogéo. Mais c'est la loi du genre de la rubrique, attachée en priorité aux questions d'actualité. La crise de Crimée était il y a peu encore à la Une des journaux. Les problèmes se sont déplacés vers l'est de l'Ukraine. À propos de la Crimée, Y. Richard montre la complexité des enjeux et le rôle des différentes parties, en premier lieu les États-Unis, l'Union Européenne et la Russie. Les réflexions de l'auteur sur les responsabilités de chacun, les risques politique et économique pris par la Russie amènent également à réfléchir audelà, sur le prolongement de la crise à l'est de l'Ukraine et au devenir des républiques ex-soviétiques. C'est ce que propose le texte de Pascal Marchand qui met en évidence l'importance des liens, notamment économiques, entre l'Ukraine et la Russie, souligne le rôle de l'Union européenne et de l'OTAN dans l'avenir du territoire et insiste sur le poids incontournable du puissant voisin russe dans la solution du conflit actuel. 